\title{
Electrochemical Performance of Low-carbon Steel in Alkaline Model Solutions Containing Hybrid Aggregates
}

\author{
D.A. Koleva ${ }^{\mathrm{a}}$, Jie Hu $\mathrm{H}^{\mathrm{a}}$, J. H. W. de Wit ${ }^{\mathrm{b}}$, N. Boshkov ${ }^{\mathrm{c}}$, \\ Ts. Radeva ${ }^{\mathrm{c}}$, V. Milkova ${ }^{\mathrm{c}}$, K.van Breugel ${ }^{\mathrm{a}}$ \\ ${ }^{\mathrm{a}, \mathrm{b}}$ Delft University of Technology, The Netherlands \\ ${ }^{a}$ Faculty CiTG, Dept. Materials \& Environment, Stevinweg 1, 2628 CN Delft \\ ${ }^{b}$ Faculty $3 \mathrm{mE}$, Dept. Surfaces \& Interfaces, Mekelweg 2, 2628 CD Delft \\ ${ }^{\mathrm{c}}$ Bulgarian Academy of Sciences, Institute of Physical Chemistry, Sofia, Bulgaria
}

\begin{abstract}
This work reports on the electrochemical performance of lowcarbon steel electrodes in model alkaline solutions in the presence of $4.9 .10^{-4} \mathrm{~g} / 1$ hybrid aggregates i.e. cement extract, containing PDADMAC (poly (diallyl, dimethyl ammonium chloride) / PAA (Poly (acrylic acid)/ PDADMAC over a $\mathrm{CaO}$ core. The main objective was to determine if the addition of hybrid aggregates will lead to increased corrosion resistance of the steel surface layers, generally formed in the hereby investigated environmental medium. Further, it was expected that when chlorides are involved, as corrosion accelerating factor, the presence of hybrid aggregates will delay corrosion initiation and therefore lead to increased corrosion resistance. This investigation forms part of a novel approach to control steel corrosion in reinforced concrete, using self-healing mechanisms. The results from this study denote for indeed superior corrosion performance of steel in chloride-free and chloride containing alkaline solution, when hybrid aggregates are involved. The mechanisms are related to increased barrier effects of the formed layer and $\mathrm{CaO}$ release from the core of the aggregates.
\end{abstract}

\section{INTRODUCTION}

This work forms part of the first initial tests in the frame of a project on self-healing corrosion damages in reinforced concrete. The main research objective is to initially improve the properties of the steel surface and the bulk cementitious matrix, by introducing polymeric micro/nano aggregates with tailored properties. Further, only in the event of detrimental external influence $\left(\right.$ as $\mathrm{CO}_{2}$ penetration and thus carbonation in the matrix, or $\mathrm{Cl}^{-}$penetration and thus localized corrosion on the steel surface) the aggregates will participate in a self-healing mechanism, by means of releasing initially incorporated in their core certain chemical substance (e.g. $\mathrm{CaO}$ ). The result will be restored alkalinity in the bulk matrix and repaired passive layer on the steel surface. Preliminary investigations were recently reported, where micelle formations (instead of the hereby discussed hybrid aggregates) were added in solutions (1) as well as already admixed, directly in reinforced cement-based materials $(2,3)$.

Since the intended for use tailored polymeric aggregates are formations, serving far different purposes, as drug delivery for example, rather then the hereby studied 
phenomena in alkaline solutions (and further in reinforced concrete respectively), the initial investigation is related to different aspects. Some of them concern: proper choice of the polymeric formations, proper choice of the substance that they carry, size, behavior in alkaline medium etc. Additionally, their addition to concrete as a "solid" electrolyte and the complexity of the involved hydration mechanisms, thus also particles/cement interactions, need to be well clarified in advance.

To this end, this preliminary research tries to answer the question if the hereby used hybrid formations (Fig.1) have any impact on corrosion behavior of steel in alkaline medium, with and without chlorides, and thus to make the path for further investigation in a real cement-based system. The initial tests show very promising results and hereby report on the electrochemical behavior of steel electrodes in cement extract, containing the hybrid aggregates.
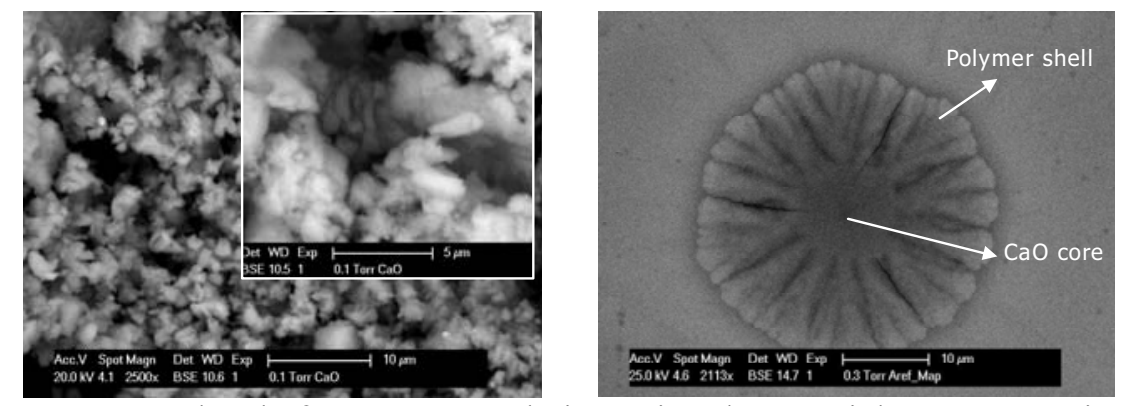

Fig.1 $\mathrm{CaO}$ crystals, before "encapsulation" in the particles; Composite (hybrid) aggregates: $\mathrm{CaO}$ core/ polymer (triple layered) shell.

\section{MATERIALS AND METHODS}

The investigated specimens were steel electrodes (low-carbon steel St37) with service area of $0.7 \mathrm{~cm}^{2}$. There was no special pretreatment, only grinding, polishing and acetone rinsing before testing were performed for each electrode. The corrosion behavior of the steel electrodes was investigated in four types of testing solutions: two controls i.e. without hybrid aggregates (form here on, for simplicity, the aggregates will be referred to as "particles") and two with particles. One of the controls and one of the particlecontaining solutions contained $\mathrm{NaCl}$ as well $(5 \%)$, as a corrosion accelerating factor. The initial (basis) solution was cement extract (CE), prepared by mixing Portland cement CEM I 42.5R and water in a ratio of $1: 1$ and filtrating the suspension after $24 \mathrm{~h}$. Thus sample designation is as follows: $\mathrm{CE}$ (CE only); CEtr.l (CE + particles); $\mathrm{CEN}(\mathrm{CE}+5 \%$ $\mathrm{NaCl})$ and $\mathrm{CENtr} .1(\mathrm{CE}+$ particles $+5 \% \mathrm{NaCl})$.

The particles, hybrid (composite) aggregates, were synthesized through a layer-bylayer adsorption of oppositely charged ployelectrolytes on $\mathrm{CaO}$ crystals (Fig.1) (PDADMAC/PAA/PDADMAC over $\mathrm{CaO}$ ). Polyelectro-lytes: poly(diallyldimethyl ammonium chloride) PDADM AC, $4.10^{-2} \mathrm{~g} / 1$; Poly(acrylic acid), sodium salt, PAA, 4.10$2 \mathrm{~g} / \mathrm{l}$. The particles concentration was $3.10^{-2} \mathrm{~g} / 1$ (demi water). In the hereby tested solutions (CE), particle concentration was $4.9 .10^{-4} \mathrm{~g} / \mathrm{l}$.

The experimental methods involved were Electrochemical Impedance Spectroscopy (EIS) and Potentio-dynamic polarization (PDP), using EcoChemie Autolab and the corresponding FRA and GPES software; SEM imaging of the steel/cement paste interface for all conditions, coupled with EDX analysis, using ESEM Philips XL30. 


\section{RESULTS AND DISCUSSION}

Before discussing the results from electrochemical tests, a brief visualization of the hybrid aggregates is presented in what follows. Figure 2 depicts the particles as received (in demi water) and after correction of the $\mathrm{pH}$ of the as received solution, initially at $\mathrm{pH}$ 11.8 (with $\mathrm{HCl}$ ) to $\mathrm{pH} 9$ and $\mathrm{pH} 3$.

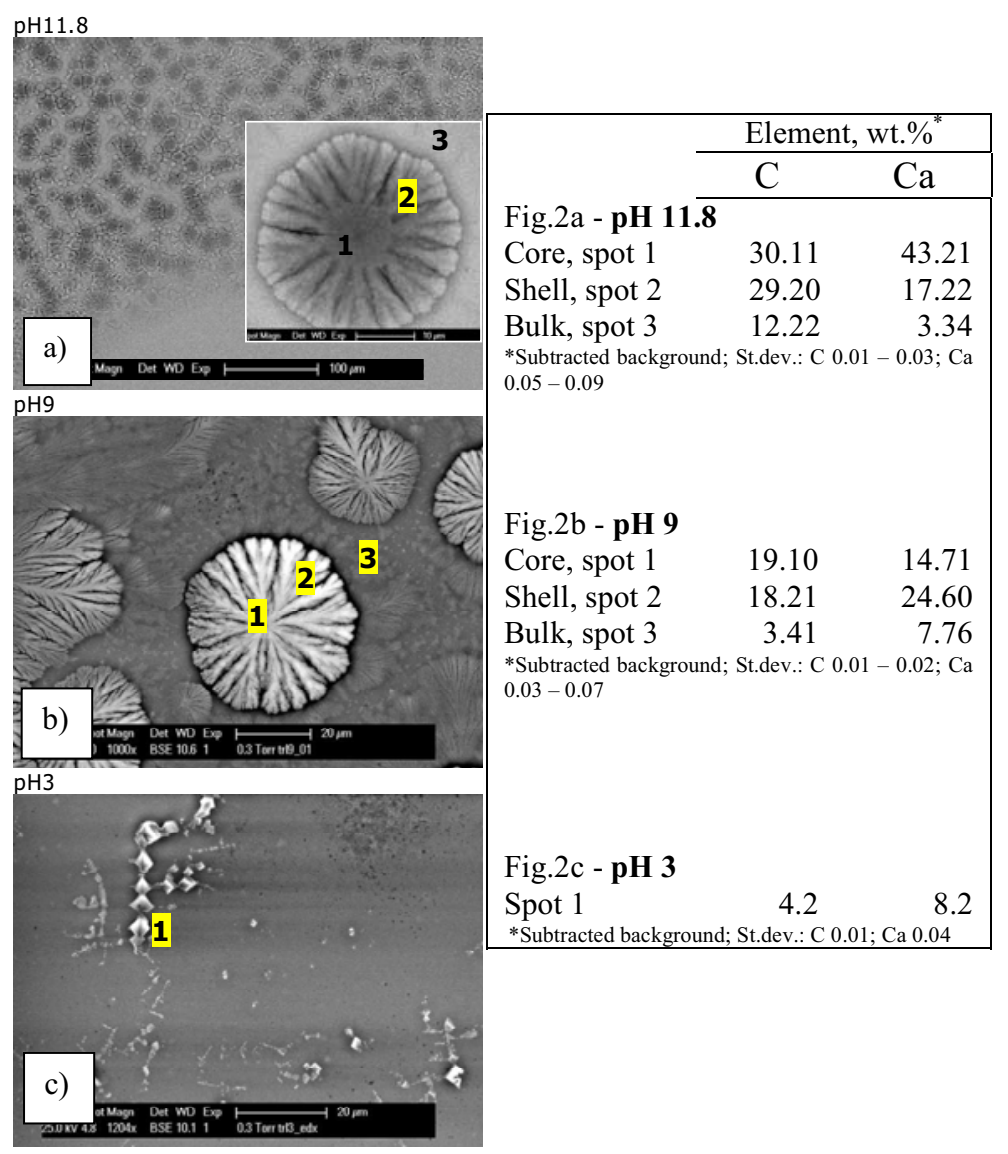

Fig.2 ESEM micrographs (left) of the hybrid aggregates as received at pH 11.8 (a); at pH 9 (b) and at $\mathrm{pH} 3$ (c); EDX analysis for $\mathrm{C}$ and $\mathrm{Ca}$ in the pointed locations (right)

The objective of this investigation was to determine if and how the morphology of the aggregates is altered within $\mathrm{pH}$ change (which would be normally the case within corrosion initiation at the steel surface) and to observe if there will be changes in the $\mathrm{CaO}$ concentration within the "core" of the particle, the "shell" and the surrounding bulk. The observations were recorded after vacuum evaporation of a drop form the solutions on a glass holder; visualization is by ESEM (in a BSE mode) and semi-quantification is derived by EDX (hereby it can be noted that EDX gives relative values, so absolute concentrations are not claimed). The evidence for higher and/or lower concentration of a certain element can be judged from the intensity of the peaks in the EDX patters. For simplicity and convenience, however, these patterns are not presented for each spot, but their corresponding values in wt.\%. In that sense, the presence of carbon denotes for the polymer, differing in amounts in the particle and in the bulk (the proximity of the 
particle); the changes in amounts of calcium denotes for $\mathrm{CaO}$ release (originally in the core) towards the shell and further in the bulk. As can be observed from Fig.2, the morphology of the particles changes within a $\mathrm{pH}$ drop from 11.8 to 9, corresponding also to a decrease of the Ca content in the "core" and increase in the "shell". Further, within a $\mathrm{pH}$ change from 9 to 3 , the polymers vanish (dissolve) and are not detected, whereas calcium-containing compounds are only detected in the bulk (the absence of polymer and presence of $\mathrm{CaO}$ crystals only were also observed after synthesis of the aggregates, within $\mathrm{pH}$ change from 11.8 down to 3 by TEM (not hereby presented)).

Further, the electrochemical behavior of steel electrodes in model solutions (with and without particles and with and without $\mathrm{NaCl}$ ) will be discussed. The measurements were performed at certain time intervals ( $1 \mathrm{~h}$ to $12 \mathrm{~h}$ and 7 days hereby presented), during which the electrodes were immersed (at OCP) in the relevant solutions. Figure 3 presents the EIS response of the control (non-corroding) groups of steel electrodes, in particles-free cement extract (Fig.3 top, denoted "control cells CE") and in particle-containing cement extract (Fig.3 bottom, denoted "control cells CEtr.l"). Similar behavior can be observed, corresponding to the passive state of all steel electrodes in this medium. What can be noted, however, is the twice higher global impedance for the CEtr.l cells (the presence of particles in the solution apparently improves the properties and corrosion resistance of the product layer). Additionally, stabilization of the passive layer in both CE and CEtr.l cases appears to be after $6 \mathrm{~h}$ of immersion (similar response for all control cells at $6 \mathrm{~h}$ and $12 \mathrm{~h}$ ), which explains the initially ( $1 \mathrm{~h}$ and $3 \mathrm{~h}$ ) lower impedance (summarized values of best fit parameters are presented further below in Table 1).
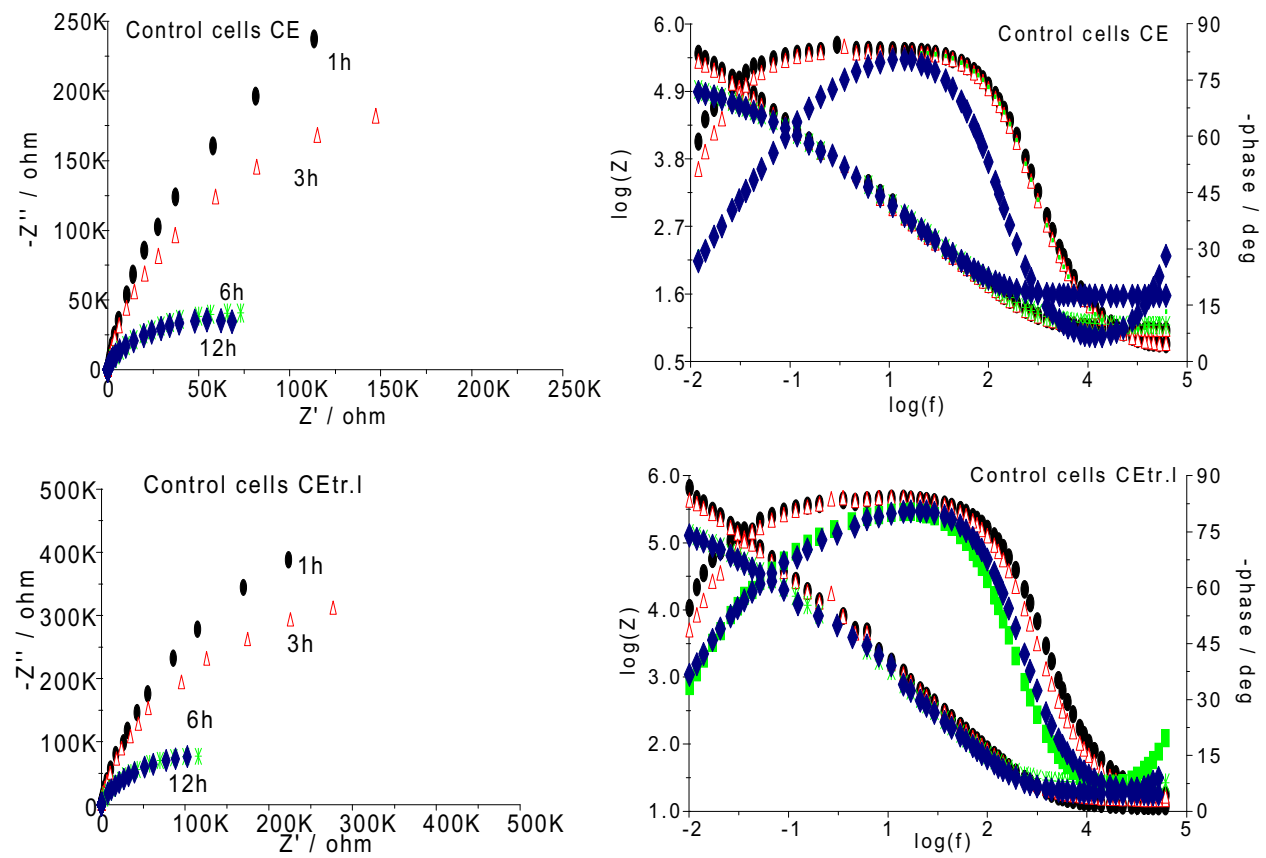

Fig.3 EIS response for control cases: CE (top) and CEtr/1 (bottom), (1h $-12 \mathrm{~h})$

As for the "corroding" cases i.e. steel conditioned in the same cement extract (with and without particles) but also in the presence of $\mathrm{NaCl}$ (added as a solid, thus $5 \%$ solution was obtained), the magnitude of impedance and corrosion resistance of both CEN (particle free) and CENtr.l (particle containing) cases is significantly lower, Fig.4. What 
can be observed, though, is again the higher magnitude of impedance in the case of CENtr.1 and even stabilization of the product layer between $6 \mathrm{~h}$ and $12 \mathrm{~h}$, whereas for the CEN case, a decreasing trend was observed (Fig. 4 bottom and top, Nyquist plots). Additionally, there is a shift of the phase angle for CENtr.1 towards lower frequency, denoted actually to increased resistance of the product layer (Fig.4, Bode plot, bottom).
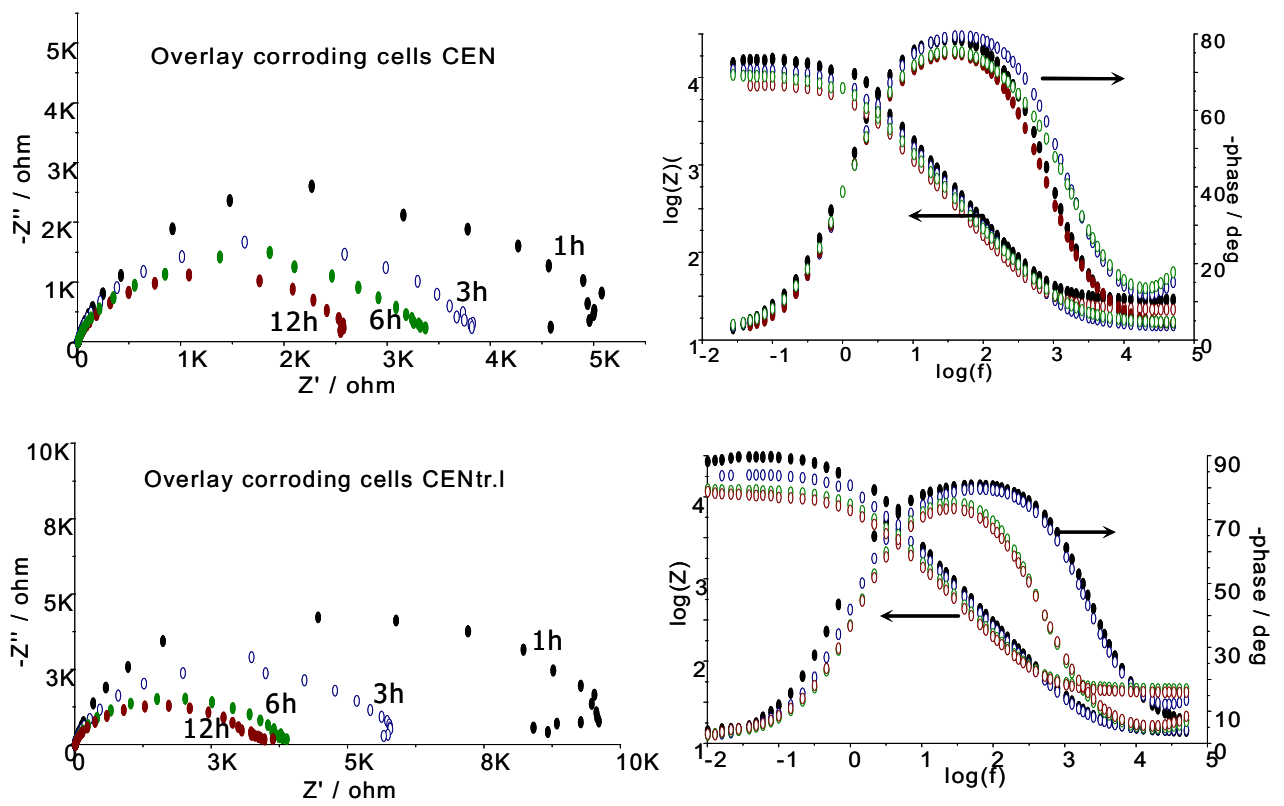

Fig.4 EIS response for corroding cases CEN (top) and CENtr/l (bottom), $(1 \mathrm{~h}-12 \mathrm{~h})$

Although the positive effect of the particles in both "control" and "corroding" cases can be clearly observed (Figs.3,4), these are not so obvious in the time intervals of $1 \mathrm{~h}$ to $12 \mathrm{~h}$. Therefore, longer treatment was considered, hereby presented as a 7 days stage. Figure 5 (top) presents a comparison of the response in the control cases at $12 \mathrm{~h}$ and 7 days, showing the increase of impedance from $12 \mathrm{~h}$ to 7 days for both $\mathrm{CE}$ and CEtr.l., however with highest impedance for the steel conditioned in particle containing solution (CEtr.1 7d). Figure 5 (bottom) depicts the comparison of corroding cells CEN and CENtr.1. An obvious difference is observed; the steel electrodes in chloride and particle containing solution presents slightly decreased magnitude of impedance from $12 \mathrm{~h}$ until 7 days of treatment, whereas the steel in chloride containing, particle-free solutions presents a significantly increased corrosion activity (impedance and phase angle drop with treatment). Table 1 presents the calculated best fit parameters; the EIS response was fitted by using (in most cases and for simplicity) an equivalent electrical circuit with one time constant only: $R_{\mathrm{el}}\left(\mathrm{Q}_{\mathrm{dl}} \mathrm{R}_{\mathrm{p}}\right)$, where the physical meaning is $\mathrm{R}_{\mathrm{el}}$ - electrolyte resistance, $\mathrm{Q}_{\mathrm{dl}}-$ double layer pseudo capacitance (a CPE was used instead of pure $\mathrm{C}$ in order to account for heterogeneity $(4,5)$ and $R_{p}$ - polarization resistance. For longer immersion intervals, an equivalent circuit with two time constants was used (denoted to the properties of the layer and to the electrochemical reaction). This latter is not hereby discussed, only summarized (re-calculated parameters are given in Table 1). 

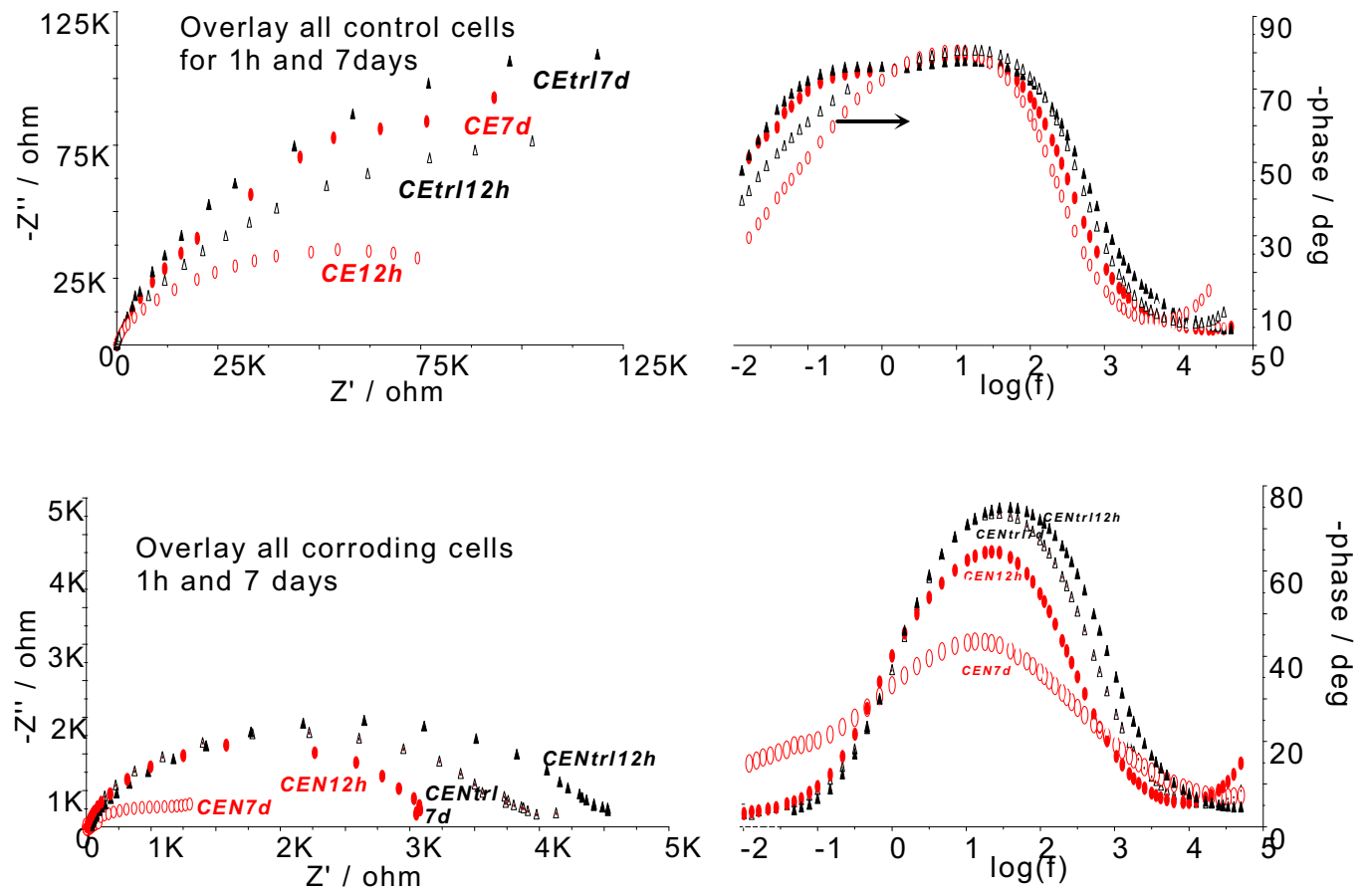

Fig.5 Overlay EIS response for control cases: CE and CEtr.1 (12h -7 days), top and for corroding cases: CEN and CENtr.1 (12h -7 days), bottom.

Table 1 Best fit parameters from EIS response Group CE (control)

\begin{tabular}{|cccc|}
\hline time & $\begin{array}{c}\mathbf{R}_{\mathbf{p}} \\
\text { kOhm.cm }\end{array}$ & $\begin{array}{c}\mathbf{Y}_{\mathbf{0}} \mathbf{Q f} \\
\mathbf{1 / O h m}\end{array}$ & $\mathbf{n}$ \\
\hline $1 \mathrm{~h}$ & 737 & $0.1338 \mathrm{e}-4$ & 0.9329 \\
$3 \mathrm{~h}$ & 429 & $0.1409 \mathrm{e}-4$ & 0.9235 \\
$6 \mathrm{~h}$ & 105 & $0.1575 \mathrm{e}-4$ & 0.8807 \\
$12 \mathrm{~h}$ & 95 & $0.1675 \mathrm{e}-4$ & 0.8775 \\
7 days & 198 & $0.2370 \mathrm{e}-4$ & 0.9126 \\
& & & \\
\hline
\end{tabular}

Group CEN (corroding)

Group CEtrl (control+hybrid aggregates)
\begin{tabular}{|ccccc}
\hline time & $\begin{array}{c}\mathbf{R}_{\mathbf{p}} \\
\text { kOhm.cm }\end{array}$ & $\begin{array}{c}\mathbf{Y}_{\mathbf{0}} \mathbf{Q f} \\
\mathbf{1 / O h m}\end{array}$ & $\mathbf{n}$ \\
\hline $1 \mathrm{~h}$ & 1090 & $0.8863 \mathrm{e}-5$ & 0.9339 \\
$3 \mathrm{~h}$ & 661 & $0.9291 \mathrm{e}-5$ & 0.9309 \\
$6 \mathrm{~h}$ & 182 & $0.1390 \mathrm{e}-4$ & 0.8936 \\
$12 \mathrm{~h}$ & 172 & $0.1963 \mathrm{e}-4$ & 0.9070 \\
7 days & 262 & $0.1383 \mathrm{e}-4$ & 0.9845 \\
\hline
\end{tabular}

\begin{tabular}{|cccc|}
\hline time & $\begin{array}{c}\mathbf{R}_{\mathbf{p}} \\
\text { kOhm.cm }\end{array}$ & $\begin{array}{c}\mathbf{Y}_{\mathbf{0}} \mathbf{Q f} \\
\mathbf{1 / O h m}\end{array}$ & $\mathbf{n}$ \\
\hline $1 \mathrm{~h}$ & 5.3 & $0.1648 \mathrm{e}-4$ & 0.9385 \\
$3 \mathrm{~h}$ & 3.8 & $0.1749 \mathrm{e}-4$ & 0.9163 \\
$6 \mathrm{~h}$ & 3.4 & $0.1419 \mathrm{e}-4$ & 0.8667 \\
$12 \mathrm{~h}$ & 2.1 & $0.2109 \mathrm{e}-4$ & 0.8952 \\
7 days & 0.8 & $0.7186 \mathrm{e}-3$ & 0.6920 \\
*incl. Warburg impedance $>1 \mathrm{mOhm}$ & \multicolumn{4}{l}{} \\
*ing
\end{tabular}

Group CENtrl (corroding+hybrid aggregates)

\begin{tabular}{|cccc}
\hline time & $\begin{array}{c}\mathbf{R}_{\mathbf{p}} \\
\text { kOhm.cm }\end{array}$ & $\begin{array}{c}\mathbf{Y}_{\mathbf{0}} \mathbf{Q f} \\
\mathbf{1} / \mathbf{O h m}\end{array}$ & $\mathbf{n}$ \\
\hline $1 \mathrm{~h}$ & 9.4 & $0.1229 \mathrm{e}-4$ & 0.9352 \\
$3 \mathrm{~h}$ & 5.8 & $0.1295 \mathrm{e}-4$ & 0.9150 \\
$6 \mathrm{~h}$ & 3.7 & $0.1626 \mathrm{e}-4$ & 0.9070 \\
$12 \mathrm{~h}$ & 3.9 & $0.7741 \mathrm{e}-5$ & 0.8172 \\
7 days & 3.6 & $0.1567 \mathrm{e}-4$ & 0.8828 \\
\hline
\end{tabular}

Finally, ESEM observation of the product layers, formed on the steel surface after 7 days of conditioning, reveals a more homogeneous and compact layer for the specimens conditioned in particle containing solutions (Fig.6c,d), whereas, the specimens treated in cement extract only, present significantly higher heterogeneity: larger isolated formations 
(Fig.6a) or steel surface, not completely covered by a product layer (Fig.6b, brighter parts in the image).
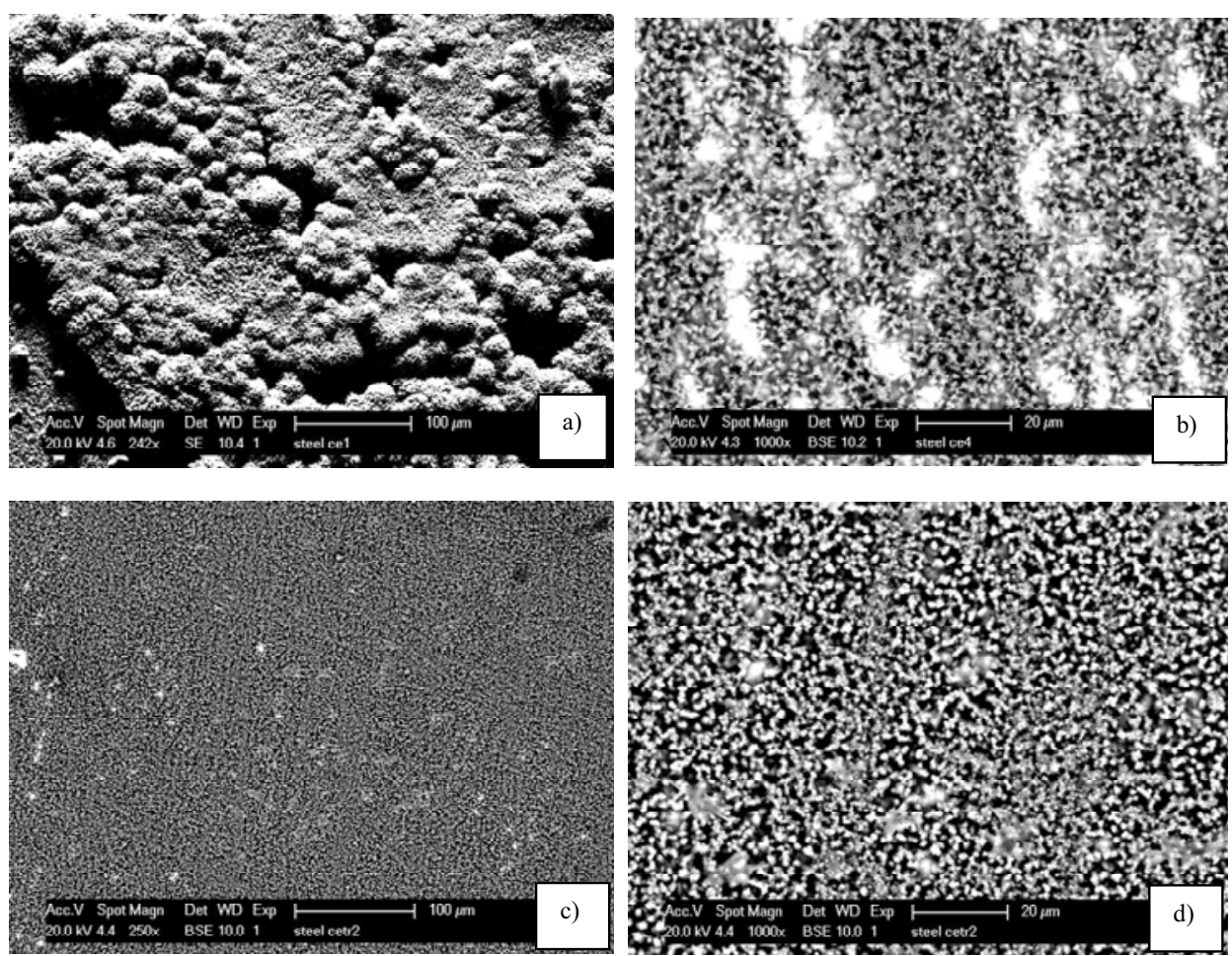

Fig.6 Product layers on the steel surface, conditioned for 7 days in cement extract only (top) and cement extract, containing hybrid aggregates (bottom)

\section{CONCLUSIONS}

In conclusion, this preliminary stage of the investigation on the influence of very low concentration, $4.9 .10^{-4} \mathrm{~g} / \mathrm{l}$, hybrid aggregates on the corrosion performance of low-carbon steel in model solutions, provides very promising results. For control alkaline model solutions (cement extract only) the corrosion resistance of the product layer on the steel surface in the presence of aggregates is apparently increased, compared to the properties of the layer on steel, conditioned in particle-free solution (Rp values in the range of 175 to $262 \mathrm{kOhm} . \mathrm{cm}^{2}$ for the former and 95 to $198 \mathrm{kOhm} . \mathrm{cm}^{2}$ for the latter case after $12 \mathrm{~h}$ and 7 days treatment). When $\mathrm{NaCl}$ is involved, as corrosion accelerator, the steel in particle containing solution, again exhibits superior performance, compared to particle-free solution (Rp values in the range of $3.6-3.9 \mathrm{kOhm} . \mathrm{cm}^{2}$ for the former and decreasing, 2.2 - $0.8 \mathrm{kOhm} . \mathrm{cm}^{2}$, for the latter case). The observed behavior is in first approximation denoted to increased barrier properties of the layers, when particles are present in the solution. Additionally, the particles probably act as nucleation sites and therefore a more uniform and compact layer is formed on the steel surface. Further, the on-going investigation will proceed with studying the electrochemical behavior of steel in model solutions within longer immersion times (in order to elucidate the involved mechanisms) and directly in reinforced concrete specimens. 


\section{REFERENCES}

1. J. Hu, D. A. Koleva, K. van Breugel, P. Petrov, J.H.W. de Wit, Preliminary Study on the Self-healing of Steel, Resulting from the Presence of Nano-aggregates in Cement Extract, $217^{\text {th }}$ Meeting of the ECS, April 25 - 30, Vancouver, Canada (2010)

2. D.A. Koleva, K.van Breugel, G.Ye, J. Zhou, G. Shamululu, E. Koenders, ACI Materials Journal, SP 267, 101-110 (2009)

3. D.A. Koleva, K.van Breugel, N. Boshkov, J.M.C.Mol, J.H.W.de Wit, ECS Transactions 25 (24), 79-85 (2010)

4. V. Feliú, J. A. González and S. Feliú S., J. Electrochem. Soc., 151, B134 (2004)

5. A. A. Sagüés, M.A. Pech-Canul and Shahid Al-Mansur A.K.M., Corros. Sci., 45, 7 (2003) 